\title{
The Measurement of Focal Diurnal Variation in the Femoral Articular Cartilage of the Knee
}

\author{
A. D. Brett ${ }^{1}$, J. C. Waterton ${ }^{2}$, S. Solloway ${ }^{1}$, J. E. Foster ${ }^{3}$, M. C. Keen ${ }^{3}$,
} S. Gandy ${ }^{3}$, B. J. Middleton ${ }^{4}$, R. A. Maciewicz ${ }^{2}$, I. Watt ${ }^{5}$, P. A. Dieppe ${ }^{6}$, and C. J. Taylor ${ }^{1}$

1 Division of Imaging Science, Manchester University, Manchester, UK

2 Cardiovascular, Metabolism \& Musculoskeletal Research Dept

AstraZeneca Pharmaceuticals, Cheshire, UK

3 Medical Physics \& Bioengineering, United Bristol Healthcare Trust, Bristol, UK

4 Safety of Medicines Dept., AstraZeneca Pharmaceuticals, Cheshire, UK

${ }^{5}$ Dept. Radiology, United Bristol Healthcare Trust, Bristol, UK

${ }^{6}$ Dept. Rheumatology, University of Bristol, Bristol, UK

\begin{abstract}
Our objective was to test the hypothesis that focal diurnal changes occur in the femoral articular cartilage of the knee in asymptomatic young adults. Six volunteers each were scanned early in the morning, and at the end of a working day spent mainly standing. This protocol was repeated on three successive weeks. Femoral cartilage segmentations were obtained using a region-growing algorithm. These segmentations then were regridded onto a 500-pixel template, and differences in the resulting thickness maps were assessed. Analysis of variance showed no significant diurnal variation in mean thickness. There were, however, statistically-significant diurnal changes in the thickness maps. Cartilage thickness decreased during the day in three specific locations which suffer the greatest biomechanical force.
\end{abstract}

\section{Introduction}

Osteoarthritis (OA) is one of the principal causes of disability in elderly people. The disease is characterised by focal structural changes, and eventual loss, of articular cartilage. The knee joint is often most severely affected, and anatomical locations suffering the highest biomechanical force are most likely to exhibit cartilage damage.

Recently, it has become possible to measure accurately and precisely the volume of the articular cartilage with MRI [13,4]. Fat-suppressed 3D spoiled gradient-echo MRI has been widely adopted, and provides good contrast with reasonable scanning times. However, because of the shape of the cartilage, manual segmentation of the images is tedious, so that for large-scale studies, it is desirable to develop semi-automatic techniques [6]. It now appears possible that MRI assessment of cartilage volume will be accurate and precise enough to measure OA disease progression and therapeutic intervention in small-scale trials, 
unlike the X-ray assessment of joint-space narrowing [3] which has poor statistical power. Before MRI can be employed in prospective controlled trials of structure-modifying (cartilage-preserving) drugs, however, it will be essential to understand potential confounding factors, such as diurnal variation.

Recently, we have described [7] a study seeking evidence for diurnal variation in the volume of the femoral articular cartilage in young adults. In that work, there was no evidence for diurnal change in cartilage volume: the average volume change (PM MINUS AM) was $-0.0010 \mathrm{~cm}^{3}(95 \%$ confidence interval -0.0945 to +0.0745$)$, a decline of $0.1 \%$. However, because OA is a disease of focal changes in the articular cartilage, we wished to determine whether regional diurnal changes could be detected in this data set. Further, we required a method of analysis which incorporated no preconceptions about the location or nature of the putative focal changes. It was therefore necessary to develop an objective method for significance testing of focal changes in a population of knees. The aims of this study were, therefore, to determine whether focal diurnal variations occur in the femoral articular cartilage of the knee.

\section{Cartilage Data}

Six volunteers (three male, three female), aged 21-25, without symptoms of any musculoskeletal disorder, were imaged in the morning (AM) at 07:45, and at 16:45 near the end of their working day (PM). Their days were spent predominantly in the laboratory, mostly standing at a bench. The imaging protocol, which is described below, was repeated on three consecutive weeks. One of the volunteers was not imaged at week two, giving a total of 34 scans.

\subsection{Imaging Protocol}

The MR parameters were [6]: field strength 1.0T (Siemens Impact); sagittal 3D spoiled gradient echo; fat saturation; TR $47 \mathrm{msec}$; TE $11 \mathrm{msec}$; flip angle 40\%; 192 phase-encoding steps; 64 slice partitions; matrix zero-filled to $256 \times 256$; FOV $140 \mathrm{~mm}$; no signal averaging, giving voxel dimensions $0.55 \times 0.55 \times 1.56$ $\mathrm{mm}$.

\subsection{Segmentation}

There are various approaches to segmentation of the articular cartilage. This study employed a data-driven segmentation method ('Tosca' version 2.3) (IBM, Winchester, UK) [5] giving rapid and precise measurements of volume and thickness assessment at sub-voxel resolution. All segmentations were performed by the same operator (J.E.F) and all the femoral cartilage was included in the analysis. To segment the femoral articular cartilage, a seed-point was placed within the area to be analysed. A region grower algorithm [15] was then started using interactively-determined values for the cartilage. The procedure was repeated for each slice in the data set. To ensure anatomical accuracy, minor adjustments to the segmented regions were required occasionally. A proportion of 
the segmentations were checked by a musculoskeletal radiologist (I.W.). J.E.F.'s intra-observer coefficient of variation with this approach is $1.5 \%$ [6]. This semiautomatic technique was considerably faster than manual segmentation. The latter can be rather operator-dependent and, because of the time-consuming nature of the operation, prone to errors due to operator fatigue. Typically a complete semi-automatic cartilage volume measurement was produced in less than an hour.

\section{Thickness Mapping}

The 3D thickness distribution of the cartilage was obtained by a generalisation of a method previously used in 2D [16]. In 3D, the method consists of three stages:

1. Each of the contours of each of the cartilage segmentations was used as input data to an automatic landmark generation algorithm. This produced the same number of corresponding points on each of the contours across all cartilage examples.

2. Each of the cartilage examples was re-sampled by linear interpolation to contain the same number of corresponding slice contours, each with the same number of defining points.

3. Thickness measurements were made at a number of corresponding points defined by a medial axis for each slice of each cartilage example.

These stages will now be described in more detail below.

\subsection{Generating Correspondence between Contours}

An automatic landmark generation algorithm [8] was used to create sets of corresponding landmarks on each of the slice contours of each of the segmented cartilage examples. This approach is possible because the topology of the slice contours does not change in a set of sagittal scans of the femoral cartilage. The algorithm is based upon a pair-wise corresponder which is used to match pairs of input shapes and measure the cost of these matches. The corresponder is used to produce a matrix of match costs between all pair combinations of the set of input shapes. The pairs with smallest matching costs are then merged to produce mean shapes. These mean shapes comprise the next level of a binary tree of merged pairs. Pairs of shapes are matched and merged at each level until a single mean shape exists at the root of the tree, the input shapes being the leaves of this tree, see Fig 1. A set of $N_{1}$ landmarks may be placed upon the mean shape at the root using, for example, a critical point detection algorithm [17]. These landmarks may then be propagated through the branches of the tree to the leaves using the fact that correspondences exists between each pair and its mean. In this way, a set of $N_{l}$ corresponding landmarks is produced on each of the input shapes. 


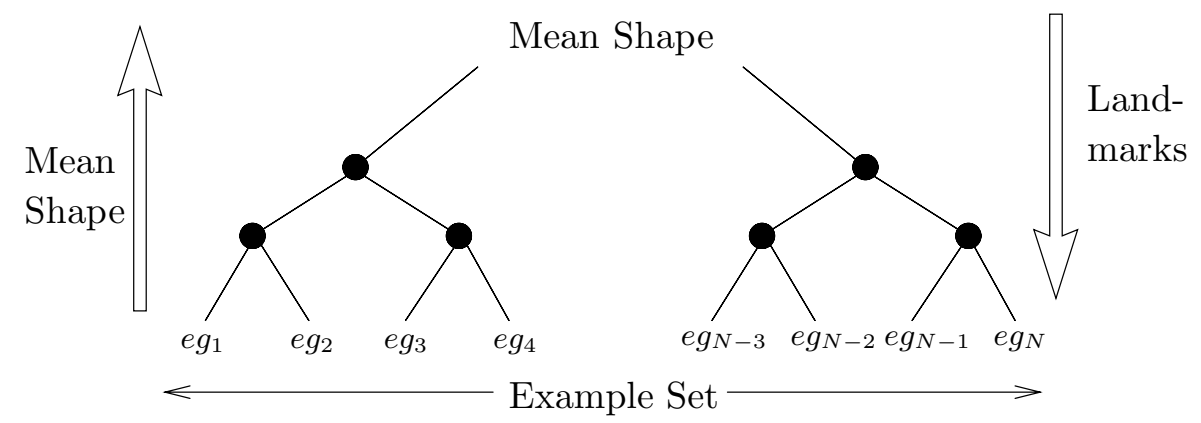

Pair-wise Corresponder

Fig. 1. A binary tree of merged shapes is produced using a pair-wise corresponder. Landmarks are propagated from a single mean shape at the root of the tree, to the example shapes at the leaves.

The pair-wise corresponder takes a pair of densely sampled shape boundaries, $\mathbf{A}$ and $\mathbf{B}$, and produces a sparse set of corresponding of point pairs on those boundaries in three stages:

1. Sparse polygonal approximations to $\mathbf{A}$ and $\mathbf{B}, \mathbf{A}^{\prime \prime}$ and $\mathbf{B}^{\prime \prime}$, are generated using the critical point detection algorithm of Zhu and Chirlian [17]. These are simply sparse representations of $\mathbf{A}$ and $\mathbf{B}$ - no correspondences are established at this stage and the two polygons will normally have differing numbers of vertices; $n_{\mathbf{A}^{\prime \prime}} \neq n_{\mathbf{B}^{\prime \prime}}$.

2. An initial estimate of corresponding sparse polygons, $\mathbf{A}^{\prime}$ and $\mathbf{B}^{\prime}$ are made. This stage uses a correspondence algorithm based on arc path length. The assumption is made that if a proportion of the boundary, say $5 \%$, of the arc path length of $\mathbf{A}$ exists between two of the vertices of $\mathbf{A}^{\prime \prime}$, then there should be $5 \%$ of the arc path length of $\mathbf{B}$ between the two points on $\mathbf{B}$ which correspond with those on $\mathbf{A}^{\prime \prime}$. A least-squared Euclidean distance metric between sets of corresponding points is used to optimise this correspondence.

3. The initial set of correspondences generated in stage 2. is refined. This stage uses a greedy optimisation scheme to modify the correspondence pairs. This produces sparse polygons $\mathbf{A}^{\prime}$ and $\mathbf{B}^{\prime}$ which are similar in shape to one another and have similar representation errors with respect to their defining boundaries $\mathbf{A}$ and $\mathbf{B}$.

\subsection{Slice Interpolation}

Correspondence between contours of each segmentation is not sufficient to produce a mapping between each segmentation example. We require correspondence between surfaces. This is achieved by linearly interpolating a new set of $N_{\mathrm{c}}$ slice 
contours between the first and last of the stack of contours representing each segmentation, see Fig 2. Each segmentation is now represented by a set of $N_{\mathrm{c}}$ contours, each having $N_{1}$ defining vertices.

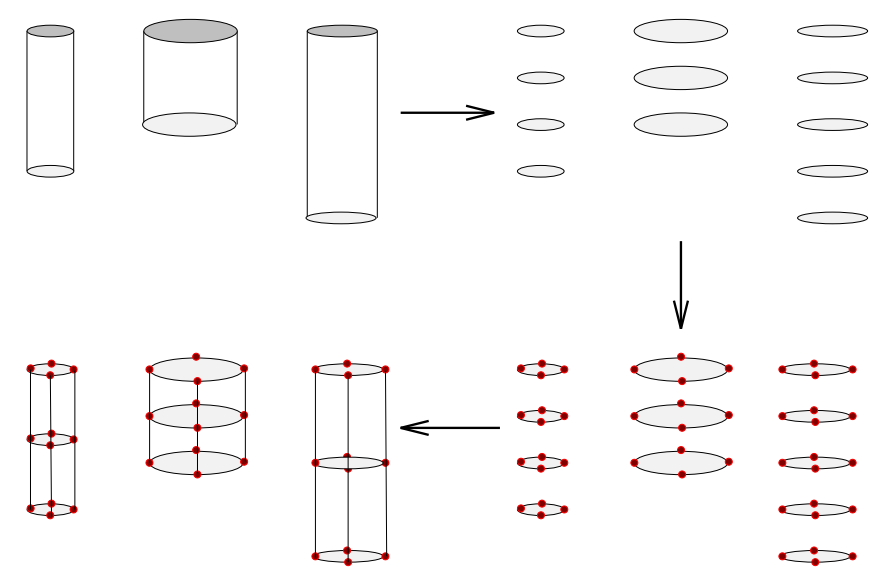

Fig. 2. Each example must have the same number of contours, each with the same number of defining vertices.

\subsection{Thickness Computation}

The medial axis of the points in each re-interpolated slice was found using the algorithm described by Shapiro [14]. A set of $N_{\mathrm{t}}$ equally-spaced points was generated along the medial axis. For each point, the thickness of the cartilage was given by the distance between the intercepts of the normal to the axis with the inner and outer surface, as shown in Fig 3.

The points, $\left(x_{j}^{\mathrm{i}}, y_{j}^{\mathrm{i}}\right)$ and $\left(y_{j}^{\mathrm{o}}, x_{j}^{\mathrm{o}}\right)$, of intersection of the normal to the $j^{\text {th }}$ point along the medial axis with the inner and outer surface respectively, are found by solving the equations,

$$
\begin{aligned}
& \left(x_{j}^{\mathrm{m}}-x_{j}^{\mathrm{i}}\right) \cos \theta+\left(y_{j}^{\mathrm{m}}-y_{j}^{\mathrm{i}}\right) \sin \theta=0 \\
& \left(x_{j}^{\mathrm{m}}-x_{j}^{\mathrm{o}}\right) \cos \theta+\left(y_{j}^{\mathrm{m}}-y_{j}^{\mathrm{o}}\right) \sin \theta=0
\end{aligned}
$$

where $j=1 \ldots N_{\mathrm{t}}$. The thickness, $t$ of the cartilage at each point is given by

$$
t_{j}=\sqrt{\left(x_{j}^{\mathrm{i}}-x_{j}^{\mathrm{o}}\right)^{2}+\left(y_{j}^{\mathrm{i}}-y_{j}^{\mathrm{o}}\right)^{2}} .
$$

This slice-by-slice method overestimates thickness where the slice plane is $<90^{\circ}$ to the surface, e.g. between the condyles: however since the thrust of our work is to measure differences in thickness, these small systematic errors should cancel. In any case most of the condylar surface, both load-bearing and 


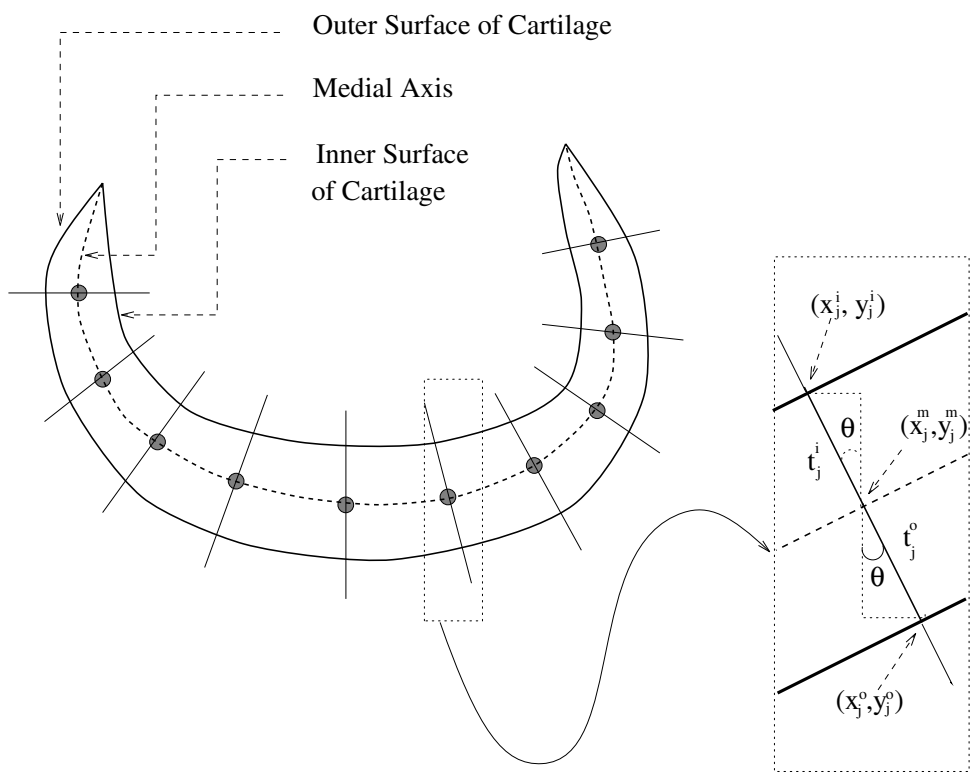

Fig. 3. Thickness measurements made in $2 \mathrm{D}$ along the medial axis of the contour defining the cartilage on one slice.

non load-bearing is very close to $90 \%$ to the sagittal plane. By measuring the thickness with respect to the medial axis, the measurements are robust to small indentations on the cartilage surfaces.

\subsection{Thickness Maps}

The resulting thickness distribution maps are analogous to those obtained by other workers using different algorithms [12,10]. Each 3D example was re-sampled to produce a correspondence of $N_{\mathrm{c}}=25$ sagittal slices with $N_{\mathrm{l}}=38$ landmarks as defining vertices. The medial axis of each slice was sampled to give $N_{\mathrm{t}}=20$ thickness values so that a $25 \times 20$ point thickness map was generated. The diurnal change for each subject on each day was represented in the difference map, PM MINUS AM. The population mean diurnal difference was obtained from the mean of all seventeen PM MINUS AM maps. Additionally, a mean thickness value was calculated from each of the 34 thickness maps.

\section{Statistical Analysis of Global Changes}

Analysis of variance was used to test the hypothesis that there was a diurnal change in the mean thickness. The data were fitted using date, time of day and gender as fixed terms and subject-within-gender as random. To judge date, time of day, their interaction and how each interacts with gender, their interaction 
with subjects-within-gender were also fitted. The reproducibility of the method was assessed from the test-retest coefficient of variation $(\mathrm{CoV})$. For each subject, $i$, the $\mathrm{CoV}$ is the standard deviation, $\sigma_{i}$, for a series of measurements on that subject, divided by the mean volume, $\mu_{i}$, for the subject. The overall testretest $\mathrm{CoV}$ for a group of $N$ subjects is then $\sqrt{\sum_{i}\left(\sigma_{i} / \mu_{i}\right)^{2} / N}$. An assessment of the ability of the method to measure small weekly changes was obtained from the sum of the week-to-week term (i.e. variance for a volunteer of a given gender on a given date) $\sigma_{\text {subj(datexsex) }}^{2}$ and the residual term $\sigma_{\text {residual }}^{2}$ in the analysis of variance: the $\mathrm{CoV}$ for a weekly difference is then

$$
\frac{\sqrt{2\left(\sigma_{\text {subj }(\text { date } \times \text { sex })}^{2}+\sigma_{\text {residual }}^{2}\right)}}{\sum_{i} \mu_{i} / N} .
$$

\section{Statistical Analysis of Regional Changes}

To test whether there was a statistically significant pattern of diurnal redistribution of the cartilage thickness, a principal component and linear discriminant analysis of the 34 thickness maps was performed. This analysis was undertaken without any prior assumptions about which locations might be most susceptible to diurnal change.

- With the assumption of identical covariance matrices for the two groups, a Principal Component Analysis (PCA) [9] on normalised thickness vectors, $\mathbf{t}$, was performed, giving a set of eigenvectors, $\mathbf{P}$.

- The original vectors were projected onto the eigenvector basis $\mathbf{P}$, giving $\mathbf{t P}$. This is a data reduction step which helps to reduce the complexity of the problem.

- A Linear Discriminant Analysis was performed on the projected vectors, using AM and PM thickness patterns as separate classes. This gave the vector, $\mathbf{d}$, which provided the best discrimination between AM and PM.

- The eigenvectors, tP, were projected onto the discriminant vector $\mathbf{d}$, giving tPd.

- The scalar values derived from this projection for each thickness map were tested to see if there were significant differences between the AM and PM maps, using the two-tailed Student's t-test.

\section{$6 \quad$ Results}

\subsection{Mean Thickness}

Analysis of variance (Table 1) also showed no evidence of diurnal variation in mean thickness. There was a trend to thicker cartilage in males than females, consistent with previous observations from MRI in young people $[2,11]$. 


\begin{tabular}{cccc}
\hline Term & Cause of variation & \multicolumn{2}{c}{ Mean thickness / mm } \\
\hline \hline Fixed terms & & Effect (95\% confidence interval) \\
\hline sex & male minus female & $0.52(1.07,-0.03)$ & $\mathrm{P}=0.059$ \\
date & week 1 vs week 2 vs week 3 & $0.11(-0.24,0.36)$ & $\mathrm{P}>0.05$ \\
time & AM minus PM & $0.02(0.22,-0.17)$ & $\mathrm{P}>0.05$ \\
\hline Random terms & & Contribution to total variance \\
\hline subj(sex) & given gender & $53.8 \%$ & $\mathrm{P}=0.036$ \\
subj(date $\times$ sex $)$ & given gender on given date & $25.5 \%$ & $\mathrm{P}=0.006$ \\
subj(time $\times$ sex $)$ & given gender at given time & $12.4 \%$ & $\mathrm{P}=0.02$ \\
residual & & $8.3 \%$ & \\
\hline
\end{tabular}

Table 1. Analysis of variance for mean thickness. For the fixed term date, data shown are for week 1 minus week 2 ; for week 1 vs week 3 , and for week 2 vs week 3 , (not shown) the effects were similar (all $\mathrm{P}>0.05$ ). Also included in the analysis of variance were the four cross terms (time $\times$ sex), (time $\times$ date), $($ date $\times$ sex $)$ and (time $\times$ date $\times$ sex). These were fixed in the analyses, and none was significantly different from zero. $\mathrm{P}$ is the probability that a term contributed to the residual a significant additional source of variance. Although the terms 'subj(date $\times$ sex)' and 'subj (time $\times$ sex)' appear significant, the significance was lost when the outlying data from volunteer at week 3 were omitted.

\subsection{Spatial Dependence of Thickness}

We sought to test the hypothesis of focal diurnal changes in regional thickness, perhaps associated with regions of mechanical loading. Fig. 4 is a colour map showing the mean of all seventeen PM MINUS AM maps. Cartilage thickness decreased during the day in specific locations: in the patellofemoral compartment, in the lateral tibiofemoral compartment, and in the medial tibiofemoral compartment. Elsewhere cartilage volume was unchanged or became thicker. Projection of the original thickness vectors, $\mathbf{t}$, onto the first two principal components of the PCA analysis showed a clear separation between the different subjects, but did not separate the AM and PM images. However a significance test of the projection $\mathbf{t P}$ of the measurement vector onto the discriminant mode, giving $\mathbf{t P d}$, showed that this diurnal change in the distribution of cartilage thickness was indeed statistically significant $(\mathrm{p}<0.05)$. This discriminant vector is shown in Fig. 5. 


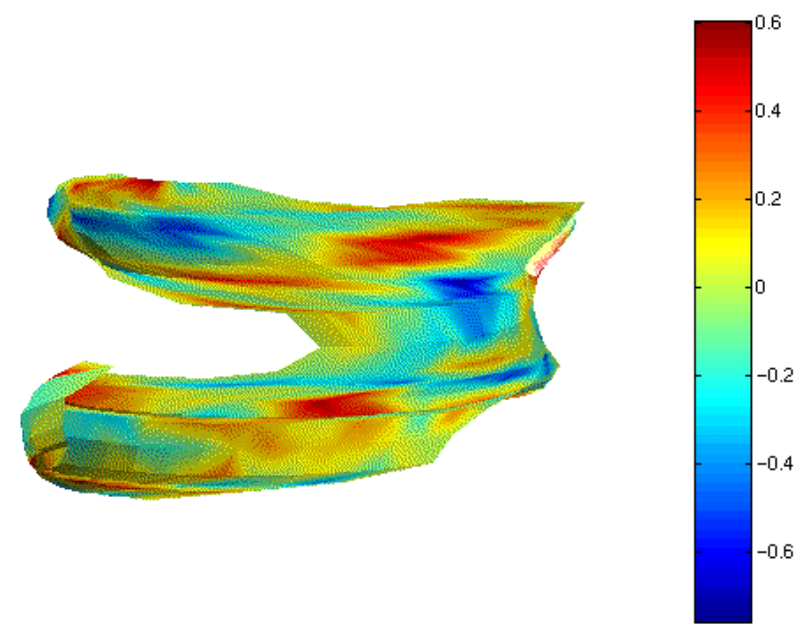

Fig. 4. Overall cartilage thickness difference map, i.e. the average of seventeen individual AM MINUS PM difference maps. The scale represents thickness change in $\mathrm{mm}$. During the day, cartilage thickness decreased by $\sim 0.5 \mathrm{~mm}$ in dark blue regions, while in red regions thickness increased by $\sim 0.5 \mathrm{~mm}$. Patellofemoral compartment is at right of figure, medial tibiofemoral compartment is bottom left in figure, lateral tibiofemoral compartment is top left.
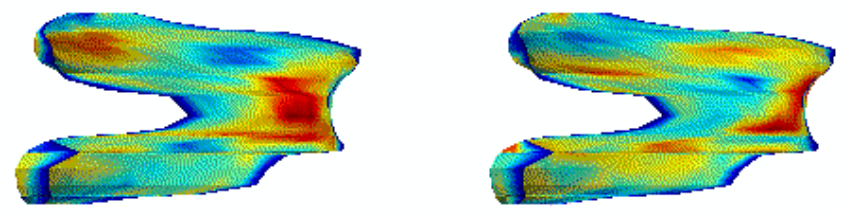

Fig. 5. Two instances of the principal components of the diurnal thickness variation. By moving along the vector which best discriminates between AM and PM measurements in the space of the principal components, we can examine the local changes to the cartilage thickness. These two figures show extremes in variation at +2 and -2 standard deviations from the mean of the cartilage thickness. The red areas are those which exhibit the greatest deviations from the mean, while the blue areas exhibit the least variation. 


\section{Conclusions}

Patients with OA lose cartilage not uniformly, but preferentially at certain locations, so that focal measurements of thickness will provide more information than volume alone, provided that good statistical power can be achieved. The method employed in this study allows multiple images from the same or different subjects to be regridded onto a standard template. Image analysis using this standard template allowed an objective evaluation of regional thickness changes common to our population, and allowed tiny physiological changes in articular cartilage thickness to be detected from data acquired using a standard clinical MRI protocol. A strength of the approach is that the evaluation is not influenced by prior expectations about which locations might be most likely to change. Nevertheless, diurnal cartilage thinning in this study was observed precisely at those sites known to be most susceptible to OA [1].

\section{References}

1. P. G. Bullough. Osteoarthritis and related disorders: pathology. In J. H. Klippe and P. A. Dieppe, editors, Rheumatology, volume 2, pages 8.8.1-8.8.8, London, 1998. Mosby Yearbook. 337

2. M. Cova, F. Frezza, I. Shariat-Razavi, M. Ukmar, R. S. P. Mucelli, and L. D. Palma. Valutazione con risonanza magnetica degli aspetti della cartilagini ialine articolari del ginocchio in funzione dell'età, del sesso e del peso corporeo. Radiol. Med., 92:171-179, 1996. 334

3. P. A. Dieppe, J. Cushnaghan, and L. Shepstone. The Bristol 'OA500' study: Progression of osteoarthritis over three years and the relationship between clinical and radiographic changes at the knee joint. Osteoarthr. Cartilage, 6:87-97, 1997. 329

4. F. Eckstein, H. Sittek, S. Milz, and M. Reiser. The morphology of articular cartilage assessed by magnetic resonance imaging. Surg. Radiol. Anat., 16:429-438, 1994. 328

5. P. J. Elliott, J. Diedrichsen, K. J. Goodson, R. Riste-Smith, and G. J. Sivewright. An object-oriented system for 3D medical image analysis. IBM Systems Journal, 35(1):5-24, 1996. 329

6. J. E. Foster, P. A. Dieppe, R. A. Maciewicz, J. Taberner, I. Watt, and J. C. Waterton. Quantification of cartilage volume and visualisation of osteoarthritis using a clinical MR system. Arthr. Rheum., 39:s170, 1996. 328, 329, 330

7. J. E. Foster, M. C. Keen, I. Watt, P. A. Dieppe, R. A. Maciewicz, J. C. Waterton, and B. J. Middleton. Measurement of human articular cartilage volume: Diurnal effects on precision. In Proc. ISMRM, 5th Meeting, page 344, Vancouver, 1997. 329

8. A. Hill, A. D. Brett, and C. J. Taylor. Automatic landmark identification using a new method of non-rigid correspondence. In J. Duncan and G. Gindi, editors, $15^{\text {th }}$ Conference on Information Processing in Medical Imaging, pages 483-488, Poulteney, VT, 1997. Springer-Verlag. 330

9. I. T. Jolliffe. Principle Component Analysis. Springer-Verlag, New York, 1986. 334

10. A. Lösch, F. Eckstein, M. Haubner, and K.-H. Englmeier. A non-invasive technique for three-dimensional assessment on articular cartilage thickness based on MRI part 1: development of a computational method. Magn. Reson. Imaging, 15:795804, 1997. 333 
11. S. Lukasz, R. Muhlbauer, S. Faber, K.-H. Englmeier, M. Reise, and F. Eckstein. Geschlechtsspezifische Analyse der Knorpelvolumina des Knieelenks - eine quantitative MRT-basierte Studie. Anat. Anz., 180(6):487-93, 1998. 334

12. C. A. McGibbon, D. E. Dupuy, W. E. Palmer, and D. E. Krebs. Cartilage and subchondral bone thickness distribution with MRI. Acad. Radiol., 5:20-25, 1998. 333

13. C. G. Peterfy, C. F. van Dijke, D. L. Janzen, C. C. Glüer, R. Namba, S. Majumdar, P. Lang, and H. K. Genant. Quantification of articular cartilage in the knee with pulsed saturation transfer subtraction and fat-suppressed MR imaging: Optimization and validation. Radiology, 192:485-491, 1994. 328

14. B. Shapiro and J. Sklansky. Skeleton generation from $\mathrm{x}, \mathrm{y}$ boundary sequences. Computer Vision, Graphics and Image Processing, 15:136-153, 1981. 332

15. G. Sivewright and P. Elliot. Interactive Region and Volume Growing in MR and CT. Medical Informatics, 19(1):71-80, 1994. 329

16. S. Solloway, C. E. Hutchinson, J. C. Waterton, and C. J. Taylor. The use of active shape models for making thickness measurements of articular cartiage from MR images. Magnetic Resonance in Medicine, 37:943-952, 1997. 330

17. P. Zhu and P. M. Chirlian. On critical point detection of digital shapes. IEEE Transactions on Pattern Analysis and Machine Intelligence, 17(8):737-748, 1995. 330,331 